\title{
Expiratory muscle pressure and breathing mechanics in chronic obstructive pulmonary disease
}

\author{
S. Yan*, C. Sinderby" ${ }^{\#}$ P. Bielen ${ }^{\top}$, J. Beck ${ }^{+}$, N. Comtois ${ }^{\#}$, P. Sliwinski
}

Expiratory muscle pressure and breathing mechanics in chronic obstructive pulmonary disease. S. Yan, C. Sinderby, P. Bielen, J. Beck, N. Comtois, P. Sliwinski. (C) ERS Journals Ltd 2000.

ABSTRACT: Expiratory muscle recruitment is common in stable chronic obstructive pulmonary disease (COPD) patients. Due to airway obstruction, there is little reason to believe that active expiration in COPD would be mechanically effective in lowering operating lung volume. The physiological significance of expiratory muscle recruitment in COPD, therefore, remains unknown. The purpose of this study was to assess, in COPD patients breathing at rest, the effect of expiratory muscle contraction on force generating ability of the diaphragm.

The force generating ability of the diaphragm was evaluated from its pressure swing $(P$ di) for a given diaphragm electrical activity $(E$ di), where $E$ di was normalized as \% of its maximal value $(P \mathrm{di} / E \mathrm{di} / E \mathrm{di}$,max $)$. Phasic expiratory muscle contraction was measured as the total expiratory rise in gastric pressure (Pga,exp.rise).

Nineteen seated patients with moderate to severe COPD, participated in the study and 10 exhibited phasic rise in $P$ ga during expiration with a mean Pga,exp.rise of $1.91 \pm 0.89 \mathrm{cmH}_{2} \mathrm{O}$. The patients were thus divided into passive expiration (PE) and active expiration (AE) groups. There was no significant difference in various lung function and breathing pattern parameters between the two groups. $P_{\mathrm{di}} / \boldsymbol{E}_{\mathrm{di}} / \boldsymbol{E}_{\mathrm{di}}$,max was $0.63 \pm 0.07$ and $0.54 \pm 0.07 \mathrm{cmH}_{2} \mathrm{O} / \%$ in $\mathrm{PE}$ and $\mathrm{AE}$ groups, respectively, and was not significantly different between each other. Compared with $\mathrm{PE}$ group, AE group not only recruited expiratory muscles, but also preferentially recruited inspiratory rib cage muscles and derecruited the diaphragm.

The results do not support a significant improvement of the force-generating ability of the diaphragm by phasic contraction of expiratory muscles at rest in chronic obstructive pulmonary disease patients.

Eur Respir J 2000; 16: 684-690.
*Meakins-Christie Laboratories, McGill University and Montreal Chest Institute, Royal Victoria Hospital, "MaisonneuveRosemont Hospital, University of Montreal, ${ }^{+}$Research Center of Ste-Justine Hospital, Montreal, Quebec, Canada, and ${ }^{\top}$ Dept of Respiratory Medicine, Institute of Tuberculosis and Lung Diseases, Warsaw, Poland.

Correspondence: P. Sliwinski Institute of Tuberculosis \& Lung Diseases Dept of Respiratory Medicine ul. Plocka 26 01138 Warsaw Poland Fax: 48226912454.

Keywords: Chronic obstructive pulmonary disease, expiratory muscles, respiratory mechanics, the diaphragm

Received: January 12000

Accepted after revision June 202000

The study was supported by the Medical Research Council of Canada, the J.T. Costello Memorial Research Fund, the Montreal Chest Institute, and the State Committee for Scientific Research of Poland. S. Yan is partly supported by the Fraser, Monat, and McPherson Scholarship, C. Sinderby is a fellow of the Parker B. Francis Foundation, and J. Beck is a FRSQ Fellow.
In a previous study by NINANE et al. [1], it was clearly indicated that many patients with chronic obstructive pulmonary disease (COPD) have rhythmic electrical activity of the transversus abdominis muscle when breathing quietly at rest. This leads to a phasic rise in abdominal pressure during expiration in these patients [2-4]. In healthy humans, expiratory muscle recruitment is considered to be mechanically beneficial to inspiration by its effect on reducing end-expiratory lung volume, lengthening the inspiratory muscles particularly the diaphragm, and therefore improving the mechanical effectiveness of the diaphragm as an inspiratory pressure pump [5].

The physiological significance of expiratory muscle contraction in COPD is not yet clear. Because of chronic airflow obstruction, many stable COPD patients have expiratory airflow limitation [6] and dynamic hyperinflation at rest $[3,4,7,8]$. This makes it unlikely for expiratory muscle recruitment in such COPD patients to be effective in lowering the end-expiratory lung volume. Based on this argument, NINANE et al. [1] predicted that contraction of expiratory muscles, such as the transversus abdominis, would contribute little to improving the force generating ability of the diaphragm in COPD. On the other hand, DoDD et al. [9] proposed that even if expiratory muscle recruitment may not effectively reduce operating lung volume in COPD, it would still be able to decrease abdominal volume and thus still put the diaphragm at longer length or better mechanical geometry by which the force production of the diaphragm can be improved.

The purpose of the present study was to directly evaluate the effect of expiratory muscle contraction on the force generating ability of the diaphragm in patients with COPD during spontaneous quiet breathing. The intensity of expiratory muscle contraction was quantified as the phasic increase in gastric pressure during expiration [3, 10-12]. The force generating ability of the diaphragm was defined in the present study as the ability of the diaphragm to generate transdiaphragmatic pressure for a given diaphragm activation, and was assessed as the ratio of peak transdiaphragmatic pressure to peak diaphragm electrical activity [13].

Methods

\section{Patients}

Nineteen male patients with moderate to severe COPD participated in the study. All patients were clinically stable 
without acute exacerbation of their respiratory symptoms for at least one month before the experiment. The patients were diagnosed as having COPD by a clear history, symptoms, and lung function tests suggesting chronic airflow obstruction as defined by American Thoracic Society (ATS) [14]. Each patient gave his informed consent to the experimental protocol that was approved by an appropriate institutional ethics committee.

\section{Measurements}

Respiratory flow was measured by a Fleisch No. 2 pneumotachograph (Fleisch, Lausanne, Switzerland) connected to a differential pressure transducer. Lung volume change was obtained by integrating the flow signal. Oesophageal and gastric pressures $(P$ oes and $P$ ga) were measured by conventional balloon-catheter systems via two separate differential pressure transducers. Transdiaphragmatic pressure $(P \mathrm{di})$ was calculated as $P$ ga- $P$ oes. Motions of the rib cage and abdomen were monitored by Respitrace bands placed over the rib cage (nipple level) and abdomen (umbilical level). Crural diaphragm electrical activity ( $E$ di) was measured by a multi-array oesophageal electrode [13, $15,16]$.

\section{Protocol}

While seated, patients breathed through a mouthpiece whilst wearing a noseclip. The patients were asked to perform three full inflations and three maximal sniff manoeuvres, these manoeuvres were used to determine the maximal $E$ di $(E$ di,max $)$ and maximal $P$ di $(P$ di,max $)$ [15]. Then, the patients returned to spontaneous breathing. During this period, they received no feedback from any source. They were left completely free to choose their own pattern of breathing and respiratory muscle recruitment. After the recorded signals became stable, the breathing signals were recorded for $5 \mathrm{~min}$.

\section{Data analysis}

The measured breathing signals were amplified, digitized, and recorded on a desk-top computer. All the signals were assemble averaged breath-by-breath and all the data analysis was performed on the averaged signals. The signals from breaths with oesophageal spasm and cough were discarded.

As shown in fig. 1, after the end of inspiration (the beginning of expiration), $P$ ga rapidly falls, suggesting inspiratory muscle relaxation. If this is followed by contraction of expiratory muscles during expiration, $P \mathrm{ga}$ rises again progressively to a peak towards the end of expiration (right arrow). Then, at the onset of inspiration, $P$ ga falls again with expansion of the abdomen, suggesting the effect of expiratory muscle relaxation. The intensity of phasic expiratory muscle contraction during expiration was therefore measured as total expiratory rise in $P$ ga $(P$ ga,exp.rise) using the $P$ ga,dip following the end of inspiration as baseline (fig. 1) [10-12].

The Edi signal processing methods and applications have been described in detail elsewhere [13, 15-18].
Briefly, the multiple array oesophageal electrode used in the present study consisted of nine parallel stainless steel rings arranged $10 \mathrm{~mm}$ apart, resulting in eight sequential signals of diaphragm electrical activity. These eight Edi signal outputs were amplified and digitized separately at $2,000 \mathrm{~Hz}$. They were filtered subsequently to minimize the influence of electrical signal contamination from external sources, electrode movement, adjacent muscle activity, or other sources of nondiaphragmatic electrical interference. As the diaphragm moves continuously with lung volume during breathing, its distance, which alters the filtering to each electrode pair, creates an artifact of Edi signal strength. To minimize this artifact, the position of the diaphragm relative to each pair of the electrode was determined using a cross-correlation technique for each $50 \mathrm{~ms}$ segment, and the signals obtained from above and below the diaphragm were subtracted. The detailed description of this "double subtraction technique" can be found elsewhere $[17,18]$. The root mean square (RMS) of the double subtracted signal was calculated and used to represent $E$ di, which, along with other breathing signals, was assemble averaged for further analysis.

The force generating ability of the diaphragm was calculated as the ratio of breathing peak $P$ di over peak $E \mathrm{di}$ amplitude. To minimize inter-subject variations of $E \mathrm{di}$ signal strength presumably related to electrode-muscle distance and local tissue filtering effect, the breathing $E \mathrm{di}$ was normalised as a percentage of $E$ di, max of that individual. Therefore, the force generating ability of the diaphragm was expressed as $P \mathrm{di} / E \mathrm{di} / E \mathrm{di}$,max $\left(\mathrm{cmH}_{2} \mathrm{O} / \%\right)$.

The results are presented as mean \pm SE. The two-sample t-test and simple correlation were used to perform the statistical inferences. $\mathrm{p}<0.05$ was taken to represent a significant difference.

\section{Results}

Nine patients showed no phasic increase in $P$ ga during expiration during the $5 \mathrm{~min}$ recording period. In contrast, the remaining ten patients had a clear progressive increase in $P$ ga during expiration. Thus, the patients were divided into two groups: an active expiration group (AE) who showed evidence of expiratory muscle contraction during expiration and a passive expiration group (PE) who showed no expiratory muscle contraction during expiration. Figure 1 shows the breathing signals for a patient from the PE group and a patient from the AE group. In all patients from the AE group, the expiratory muscle recruitment during expiration was consistent and was seen in every breath with little variation. The mean $P$ ga,exp.rise of the group was $1.91 \pm 0.89 \mathrm{cmH}_{2} \mathrm{O}$ with a range of $0.82-3.81 \mathrm{cmH}_{2} \mathrm{O}$ in different patients.

Table 1 shows the anthropometric and pulmonary function data for the two groups. The age, body height and weight, forced expiratory volume in one second (FEV1) forced vital capacity (FVC), static lung volumes, airway resistance, dynamic lung compliance, and resting blood gases were similar in the two groups. The only exception was dynamic intrinsic positive end-expiratory pressure (PEEPi,dyn), which was statistically higher in the $\mathrm{PE}$ than in the AE group. However, the difference in PEEP $i$,dyn between the two groups $(1.09 \pm 0.36$ versus $0.49 \pm 0.12 \mathrm{cmH}_{2} \mathrm{O}$ in $\mathrm{PE}$ and $\mathrm{AE}$ groups, respectively) 
a)

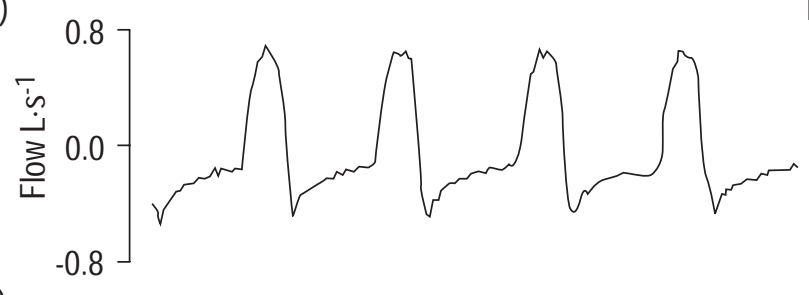

c)

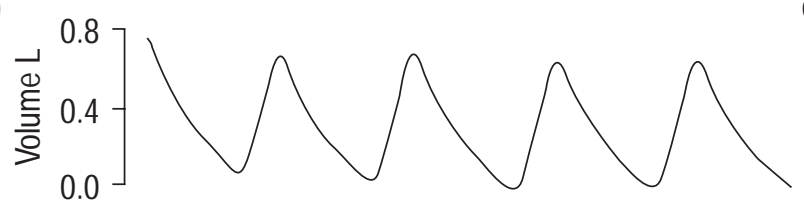

e)

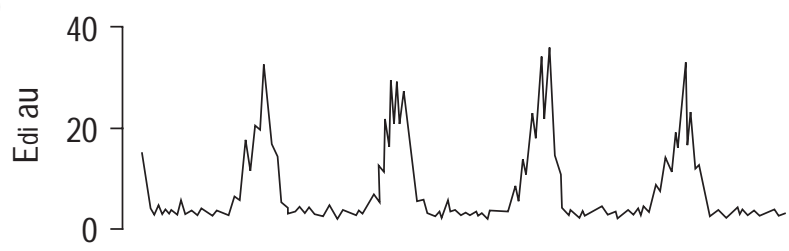

g)

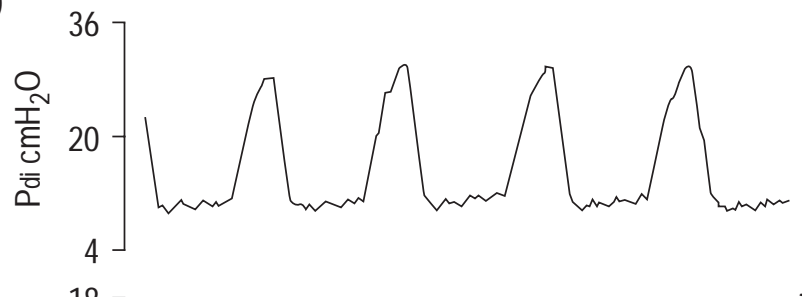

b)

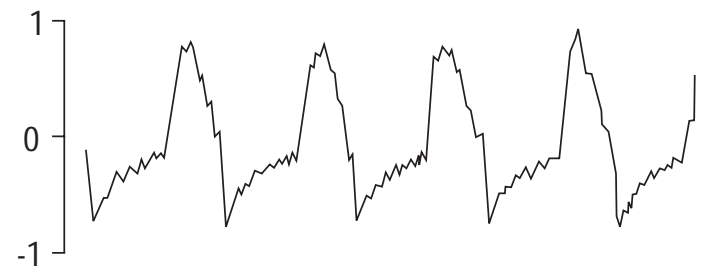

d)

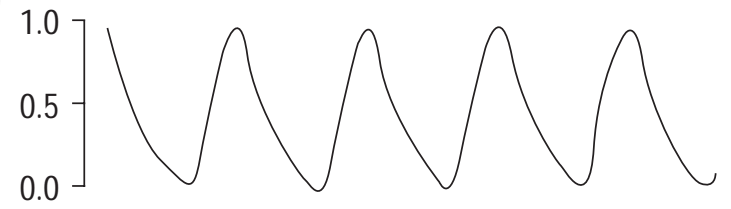

f)

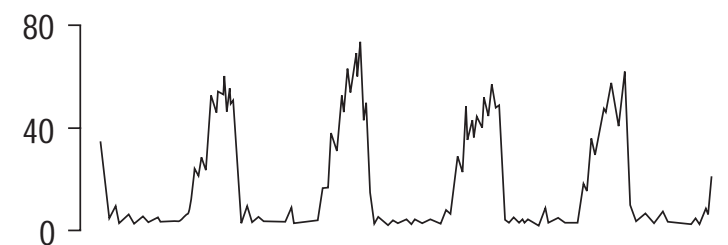

h)

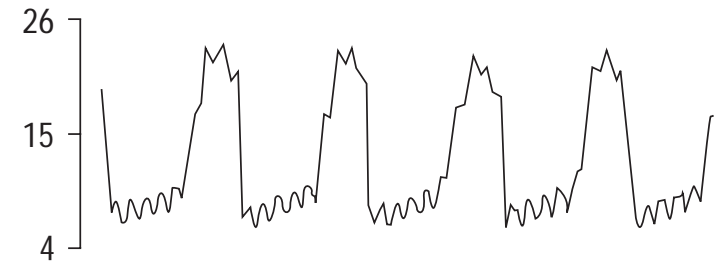

j)

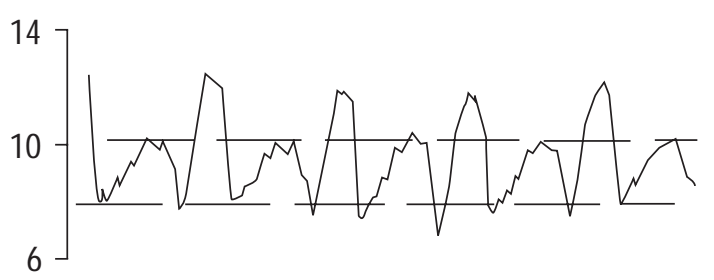

I)

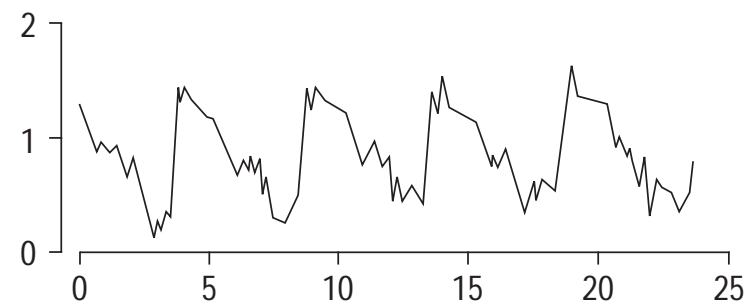

Time (s)

Fig. 1. - Records of a, b) flow; c, d) volume; e, f) diaphragm electromyogram $(E \mathrm{di}) ; \mathrm{g}, \mathrm{h})$ transdiaphragmatic pressure $(P$ di $)$; i, j) gastric pressure $(P$ ga); and $\mathrm{k}, \mathrm{l}$ ) abdominal displacement $(\mathrm{AB})$, from a patient without expiratory muscle contraction (passive expiration; a, $\mathrm{c}$, e, g, i, k) and a patient with expiratory muscle contraction (active expiration; $\mathrm{b}, \mathrm{d}, \mathrm{f}, \mathrm{h}, \mathrm{j}, \mathrm{l}$ ) during expiration; au: arbitary units. The right column shows the gradual expiratory rise in $P$ ga $(P$ ga,exp.rise $)$ from shortly after inspiration towards the end of expiration. $P$ ga,exp.rise was measured as peak expiratory $P$ ga $(--->)$ relative to the lowest $P$ ga after inspiration $(\longrightarrow)$, as indicated by the two parallel dashed lines.

was quite small. Figure 2 shows that the breathing pattern parameters, including tidal volume, breathing frequency, minute ventilation, duty cycle, and mean and peak inspiratory flow were all similar in the PE and AE groups.

Table 2 shows the mechanical and electrical activities of the diaphragm in the PE and the AE groups. Pdi,max was similar in the two groups. During quiet breathing, for a similar ventilation (fig. 2). Edi of PE group accounted for $29.1 \%$ of their Edi,max, which was significantly higher than the equivalent ratio in the AE group (20.3\%). This resulted in a significantly greater $P$ di swing during tidal breathing and $P \mathrm{di} / P$ di,max ratio in the $P E$ than in the $\mathrm{AE}$ group. In spite of these differences, $P \mathrm{di} / E \mathrm{di} / E \mathrm{di}$,max ratio, which is the parameter used to reflect the force generating ability of the diaphragm, did not differ significantly in the two groups. The corresponding $P \mathrm{di} / E \mathrm{di} / E \mathrm{di}, \max$ ratios in $\mathrm{PE}$ and $\mathrm{AE}$ groups were $0.63 \pm 0.07$ and $0.54 \pm 0.07$ $\mathrm{cmH}_{2} \mathrm{O} / \%$, respectively. 
Table 1. - Anthropometric and lung function results

\begin{tabular}{|c|c|c|}
\hline & $\begin{array}{l}\text { Passive expiration } \\
\text { group }\end{array}$ & $\begin{array}{l}\text { Active expiration } \\
\text { group }\end{array}$ \\
\hline Subjects $n$ & 9 & 10 \\
\hline Age yr & $60.1 \pm 2.5$ & $65.6 \pm 1.3$ \\
\hline Height $\mathrm{cm}$ & $171.4 \pm 3.4$ & $170.3 \pm 2.2$ \\
\hline Weight $\mathrm{kg}$ & $86.8 \pm 4.4$ & $80.7 \pm 5.4$ \\
\hline FEV1 L & $\begin{array}{c}1.10 \pm 0.13 \\
(35.1 \pm 3.3)\end{array}$ & $\begin{array}{c}1.16 \pm 0.08 \\
(37.2 \pm 2.5)\end{array}$ \\
\hline FVC L & $\begin{array}{c}3.14 \pm 0.39 \\
(76.6 \pm 8.3)\end{array}$ & $\begin{array}{c}3.05 \pm 0.22 \\
(70.1 \pm 8.03)\end{array}$ \\
\hline FEV1/FVC \% & $36.3 \pm 2.6$ & $38.7 \pm 2.8$ \\
\hline TLC L & $\begin{array}{r}7.74 \pm 0.69 \\
(118.3 \pm 9.8)\end{array}$ & $\begin{array}{c}6.86 \pm 0.43 \\
(105.3 \pm 6.2)\end{array}$ \\
\hline FRC L & $\begin{array}{c}5.58 \pm 0.46 \\
(162.5 \pm 12.9)\end{array}$ & $\begin{array}{r}5.05 \pm 0.30 \\
(145.1 \pm 8.7)\end{array}$ \\
\hline RV L & $\begin{array}{c}4.58 \pm 0.42 \\
(198.6 \pm 18.6)\end{array}$ & $\begin{array}{c}3.91 \pm 0.31 \\
(160.5 \pm 12.9)\end{array}$ \\
\hline Raw $\mathrm{cmH}_{2} \mathrm{O} \cdot \mathrm{L} \cdot \mathrm{s}^{-1}$ & $7.8 \pm 1.3$ & $6.6 \pm 0.8$ \\
\hline$C \mathrm{~L}$,dyn $\mathrm{L} \cdot \mathrm{cmH}_{2} \mathrm{O}$ & $0.16 \pm 0.003$ & $0.14 \pm 0.02$ \\
\hline PEEPi $\mathrm{cmH}_{2} \mathrm{O}$ & $1.09 \pm 0.36$ & $0.49 \pm 0.12$ \\
\hline${\mathrm{Pa}, \mathrm{O}_{2} \mathrm{mmHg}}$ & $51.9 \pm 2.6$ & $53.5 \pm 3.3$ \\
\hline 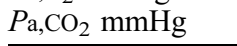 & $49.0 \pm 2.5$ & $47.0 \pm 1.4$ \\
\hline
\end{tabular}

Data are mean $\pm \mathrm{SE}$ (\% predicted). FEV1: forced expiratory volume in one second; FVC: forced vital capacity; TLC: total lung capacity; FRC: functional residual capacity; RV: residual value; $R$ aw: airway resistance; $C \mathrm{~L}$,dyn: dynamic compliance (change in lung volume/change in oesophageal pressure between beginning and end of inspiratory flow). PEEPi: intrinsic positive end-expiratory pressure (fall in oesophageal pressure before onset of inspiratory flow corrected by subtraction of any expiratory rise in gastric pressure [10]); $\mathrm{Pa}, \mathrm{O}_{2}$ : oxygen arterial tension; $\mathrm{Pa}, \mathrm{CO}_{2}$ : carbon dioxide arterial tension.

\section{Discussion}

In the present study, phasic expiratory muscle contraction was quantified by measuring the amplitude of expiratory rise in $P$ ga. The force generating ability of the diaphragm was evaluated as the $P$ di generated for a given diaphragm electrical activity. It was demonstrated that in patients with stable COPD during quiet breathing, $P \mathrm{di} / E \mathrm{di} /$ $E$ di,max ratio was independent of whether or not there was a phasic expiratory rise in $\mathrm{Pga}$, and therefore confirmed that under the experimental conditions, expiratory muscle contraction did not improve the force generating ability of the diaphragm in these patients.

Critique of the method to quantify expiratory muscle contraction

Previously, NINANE et al. [1] evaluated abdominal muscle recruitment during quiet breathing in COPD patients by inserting needle electrodes into the abdominal muscles to record their electrical activities. In the current study, this approach to quantify expiratory muscle contraction was not used for the reasons that follow. 1) Measurement of abdominal muscle electromyogram by needle electrode is an invasive technique that requires considerable experience and monitoring facilities, such ultrasonography. 2) Although PARTHASARATHY et al. [19] have recently shown a good agreement between transversus abdominis electromyogram and expiratory rise in $P$ ga in healthy subjects, the exact relationship between expiratory muscle mechanical output (expiratory muscle force and/or shortening) and its activation remains unknown for COPD patients. The aim of the present
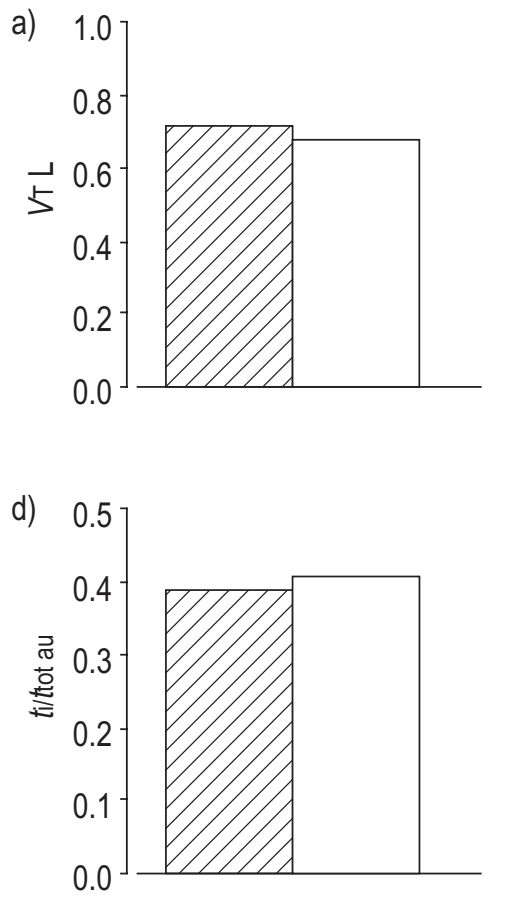
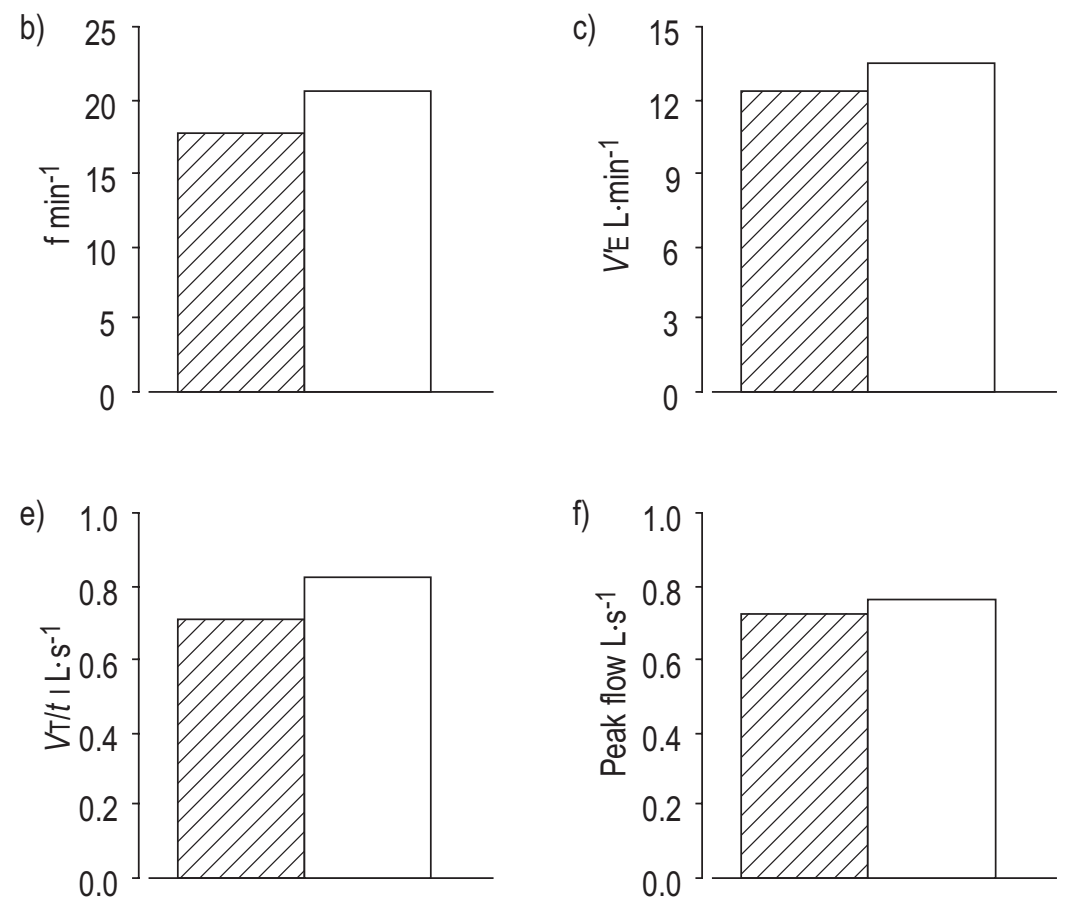

Fig. 2. - Breathing pattern parameters a) tidal volume $(V \mathrm{~T})$; b) breathing frequency (f); c) minute ventilation $V^{\prime} \mathrm{E}$; d) ratio of inspiratory time to total breathing cycle time $(t \mathrm{t} / t$ tot $)$; e) mean inspiratory flow $(V \mathrm{~T} / t \mathrm{t})$; f) peak inspiratory flow, in passive expiration $(\mathbb{Z})$ and active expiration $(\square)$. Results shown are mean \pm SE. 
Table 2. - Diaphragm electrical activity (Edi) and contractile function

\begin{tabular}{lcc}
\hline & $\begin{array}{c}\text { Passive expiration } \\
\text { group }\end{array}$ & $\begin{array}{c}\text { Active expiration } \\
\text { group }\end{array}$ \\
\hline$P \mathrm{di} \mathrm{cmH}_{2} \mathrm{O}$ & $18.6 \pm 1.2$ & $10.1 \pm 0.8$ \\
$P \mathrm{di}, \max \mathrm{cmH}_{2} \mathrm{O}$ & $100.8 \pm 8.7$ & $86.4 \pm 8.1$ \\
$P \mathrm{di} / P \mathrm{di}, \max \%$ & $19.8 \pm 2.1$ & $13.0 \pm 1.6$ \\
$E \mathrm{di} \mathrm{au}$ & $52.3 \pm 5.3$ & $40.2 \pm 5.0$ \\
$E \mathrm{di}, \max$ au & $186.5 \pm 18.1$ & $200.9 \pm 16.7$ \\
$E \mathrm{di} / E \mathrm{di}, \max \%$ & $29.1 \pm 2.9$ & $20.3 \pm 2.1$ \\
$P \mathrm{di} /(E \mathrm{di} / E \mathrm{di}, \max )$ & & \\
$\mathrm{cm} \mathrm{H}_{2} \mathrm{O} / \%$ & $0.63 \pm 0.07$ & $0.54 \pm 0.07$ \\
\hline
\end{tabular}

Values presented as mean \pm SE. $P$ di: diastolic pressure; $E$ di,max: maximum $E \mathrm{di}$; au: arbitary units. p $<0.05$ when compared between passive and active groups.

study was to assess the mechanical effect of expiratory muscle contraction, which, it is assumed, should be directly related to the mechanical output rather than activation of expiratory muscles.

$P$ ga,exp.rise has been used by several groups $[10-12,20]$ to correct the influence of expiratory muscle contraction on dynamic measurement of PEEPi during spontaneous breathing, and the results have shown that such a correction is usually successful. These studies suggest $P$ ga,exp.rise to be a reliable index of the mechanical output of the abdominal muscles, and to some extent, of the expiratory muscles as a whole, assuming that the diaphragm is relaxed and serves largely as a passive structure during expiration [10]. The fall in $P$ ga $\left(P_{\text {ga,ins.fall }}\right)$ at the beginning of inspiration (fig. 1) was not used to represent expiratory muscle pressure [21]. At the onset of inspiration, $P$ ga,ins.fall is presumably affected not only by relaxation of expiratory muscles, but also by contraction of rib cage inspiratory muscles and the diaphragm, which amplify and diminish the amplitude of $P$ ga,ins.fall, respectively. The net amplitude of $P$ ga,ins.fall is thus dependent on the unknown relative magnitudes of these three separate events. Indeed, as shown recently, there was a poor correlation between Pga,ins.fall and transversus abdominis muscle electrical activity [19]. One concern of measuring Pga,exp.rise would be that the existence of post inspiratory inspiratory muscle activity may affect the accuracy of its determination. However, both $E$ di and $P$ di had returned to baseline where the reference point of $P$ ga,exp.rise ( $P$ ga,dip after inspiration, the solid arrow in fig. 1 ) was determined. It is therefore unlikely that $P$ ga,exp.rise was influenced by post inspiratory muscle activity.

\section{Effect of expiratory muscle pressure on diaphragm mechanical function}

The presented results do not favour a significant improvement of the force-generating ability of the diaphragm by expiratory muscle contraction at rest in COPD. One may argue, however, that the $P \mathrm{di} / E \mathrm{di} / E \mathrm{di}$,max ratio in the $\mathrm{AE}$ group could have been much lower than the measured value had expiratory muscles not been recruited. The PE and AE groups in the present study had similar lung function and anthropometric data. $P$ di,max was also similar in the two groups. Therefore, diaphragm intrinsic factors, such as chronic diaphragm weakness, were unlikely to affect the interpretation of the results, and there was no reason to suspect a much lower $P \mathrm{di} / E \mathrm{di} /$ $E$ di,max ratio in $\mathrm{AE}$ group without expiratory muscle contraction. In fact, chronic diaphragm fatigue probably does not exist in stable COPD patients [22]. Peak inspiratory flow was $<0.8 \mathrm{~L} \cdot \mathrm{s}^{-1}$ and was comparable in the $\mathrm{PE}$ and $\mathrm{AE}$ groups. It has been shown that the relationship between $P$ di and $E$ di remains constant when inspiratory flow changes $0.1-1.4 \mathrm{~L} \cdot \mathrm{s}^{-1}[23]$, and therefore it is believed that inspiratory flow rate did not play a significant role in the present study. Hence, the PE and AE groups were comparable in terms of the effect of active expiration on force generating ability of the diaphragm.

Under normal conditions, contraction of expiratory muscles increases expiratory flow and thus decreases endexpiratory lung volume. This may assist the inspiratory muscles through the following mechanisms. First, inspiratory muscles are lengthened by contracting at a lower lung volume, resulting in an improved ability to generate force (better position on the length-tension relationship) [5]. Second, starting inspiration below the relaxation volume of the respiratory system reduces the elastic work of inspiration by partitioning it just above and below functional residual capacity (FRC) [24]. Third, relaxation of expiratory muscles allows the elastic recoil pressure of the respiratory system below FRC to be released, which may serve as the initial inspiratory pressure, independent of inspiratory muscle activity [25]. Therefore, expiratory muscle recruitment is mechanically beneficial to breathing in healthy subjects who, however, usually do not recruit their expiratory muscles to assist inspiration during normal resting breathing, at least in the upright sitting posture.

All these beneficial effects on breathing mechanics depend on the expiratory muscle contraction-induced fall in end-expiratory lung volume. The second and third mechanisms also require an operating lung volume below the relaxation volume of the respiratory system. If the fall in end-expiratory lung volume is prevented, the effects of expiratory muscles in assisting inspiration will be lost. This leads to the hypothesis that the beneficial effects may not apply for patients with airway obstruction because in this population, expiratory muscle contraction is unlikely to be effective in lowering end-expiratory lung volume. Indeed, using an expiratory negative pressure technique, ELTAYARA et al. [6] confirmed the presence of expiratory airflow limitation during quiet breathing in $\sim 60 \%$ of patients with stable COPD. HALUSZKA et al. [7] reported the presence of dynamic hyperinflation at rest in over two-thirds of patients with stable COPD. These observations suggest that expiratory airflow limitation is quite common in stable COPD patients at rest. It can be assumed that there would be little room left for such individuals to further reduce end-expiratory lung volume by any significant amount by active expiration during spontaneous breathing. It could be argued, though, that in COPD, the reduced abdominal volume due to expiratory muscle contraction would still lengthen the diaphragm fibres and improve its mechanical advantage, independent of lung volume [9]. The present results showed that the $P$ di swing for a given Edi was similar in the PE and AE groups, suggesting a lack of a significant effect of expiratory muscle contraction on improving the force generating ability of the diaphragm in COPD patients. These results are consistent with our expectations. In fact, 
in spontaneously breathing COPD patients, the phasic electrical activity of transversus abdominis had already ceased when inspiratory muscle electrical activity started to appear at end expiration [1]. There was little overlap between these two events. In other words, the diaphragm began to contract after the abdomen started to return rapidly to its relaxed configuration. The diaphragm was therefore, most likely to contract at a similar length regardless of expiratory muscle contraction and abdominal volume during expiration in COPD.

\section{Respiratory muscle control at rest in chronic obstructive pulmonary disease}

SINDERBY et al. [15] have recently shown that during resting breathing, $E$ di $/ E$ di,max was markedly higher in severe COPD patients $(42 \%)$ than in healthy subjects $(8 \%)$, suggesting a significantly reduced diaphragm activation reserve in COPD patients. The present results (table 2) are consistent with these observations, although $E \mathrm{di} / E \mathrm{di}$,max was slightly lower in the present patients ( 29 and 20 for the PE and AE groups, respectively), probably due to the patients having less severe disease. It was also found that the $E \mathrm{di} / E \mathrm{di}$,max ratio was significantly lower in the $\mathrm{AE}$ than in the PE group, resulting in a significantly lower $P$ di $/ P$ di,max ratio and $P$ di swing in the AE group at rest (table 2). The PE and AE groups had comparable spirometric function, airway resistance (Raw) and dynamic lung compliance $(C \mathrm{~L}, \mathrm{dyn})$, suggesting similar mechanical loads to inspiration in the two groups. It can be predicted that for the same ventilation and breathing pattern, lower diaphragm activation and pressure contribution in $\mathrm{AE}$ group must have demanded greater activation and pressure contribution from the inspiratory rib cage muscles.

MACKLEM et al. [26] originally used the slopes of the plot of pleural pressure and abdominal pressure determined at the beginning and end of inspiratory flow to partition pressure contributions between different inspiratory muscle groups. In this analysis, a greater slope of this plot indicates proportionately greater pressure contribution from the inspiratory rib cage muscles. This approach is now used to analyse the results. In figure 3 , $P$ oes values obtained at the beginning and end of inspiratory flow were plotted against the corresponding $P$ ga measurements. To minimize the effect of expiratory muscle contraction on this plot [26], for the AE group, the value of $P$ ga in the $P$ ga dip after the end of inspiratory effort (solid arrow in fig. 1) was used to represent the $P$ ga at the beginning of inspiration. For statistical convenience, the counterclockwise angle between the horizontal axis and the inspiratory $P_{\text {oes }}-P$ ga plot was calculated. The angle of $65.2 \pm 6.7$ degrees in the AE group was significantly greater than the equivalent angle of $34.8 \pm 5.8$ degrees in the PE group. This analysis confirms that the AE group recruited less diaphragm but more inspiratory rib cage muscles to produce a similar ventilation.

NINANE et al. [1] found an inverse relationship between phasic transversus abdominis muscle electrical activity and patients' FEV1. A similar relation was found between $P$ ga,exp.rise and FEV1 even within the AE group alone, probably due to the difference in methods for inferring expiratory muscle recruitment. The presented results,

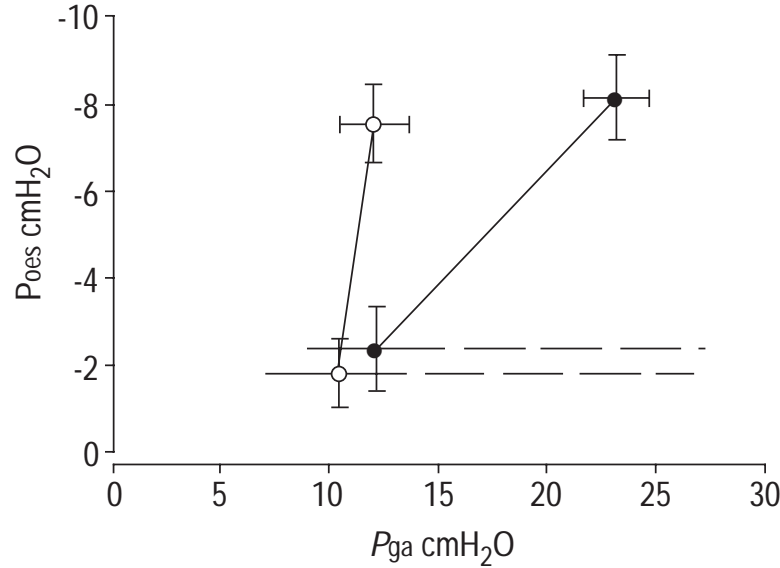

Fig. 3. - Inspiratory oesophageal pressure $\left(P_{\text {oes }}\right)$ at the beginning and end of inspiratory flow plotted against the respective values of gastric pressure $(P \mathrm{ga})$ in passive exporting $\left({ }^{\circ}\right)$ and active expiration groups (O). For each relationship, the lower left point indicates the beginning of inspiratory flow, and the higher right point the end of inspiratory flow. The angle in degrees between each inspiratory $P_{\text {oes}}-P$ ga relationship and $\mathrm{x}$-axis is $65.2 \pm 5.8^{\circ}$ and $34.8 \pm 5.8^{\circ}$. To eliminate the influence of expiratory muscle contraction on the measurement of inspiratory $P_{\text {oes- }}$ $P$ ga relationship, in the active expiration group, the lowest $P$ ga value immediately after inspiration (fig. 1, solid arrow) was used to represent the $P$ ga at the beginning of inspiration.

showing the same FEV1 in the PE and AE groups, may suggest that there are probably other factors that determine whether or not a COPD patient recruits expiratory muscles at rest. It was not the purpose of the current study to explore the mechanisms of expiratory muscle recruitment in COPD. The results, however, imply that recruitment of expiratory muscles in COPD may involve respiratory centre integrated neurocontrol mechanisms that are not limited to control of expiratory muscles alone. Indeed, the AE group also had greater recruitment of inspiratory rib cage muscles but less recruitment of the diaphragm than the PE group. This suggests that in COPD patients breathing at rest, control of nondiaphragmatic respiratory muscles, both inspiratory and expiratory, may to some extent be coupled to each other.

In summary, the present study indicated that in stable COPD patients breathing at rest, peak $P$ di swing for a given $E$ di, a parameter reflecting the contractile properties of the diaphragm, was similar irrespective of whether or not expiratory muscles contracted phasically during expiration. These results suggest no significant effect of expiratory muscle recruitment on improving the force generating ability of the diaphragm during quiet breathing in these patients. On the other hand, expiratory muscle contraction must consume additional oxygen supply, which is limited in COPD patients. In addition, it has recently been shown that in healthy humans, exertional dyspnoea was explained independently by the magnitude of expiratory pressure [27]. It is possible that expiratory muscle recruitment may also contribute to chronic dyspnoea in COPD patients.

Additional research is needed to investigate the impact of expiratory muscle recruitment on breathing in chronic obstructive pulmonary disease. The design of chronic obstructive pulmonary disease rehabilitation programmes, such as breathing training, may benefit from this line of research. 
Acknowledgements. The authors thank P.T. Macklem for his helpful comments to the results.

\section{References}

1. Ninane V, Rypens F, Yernault JC, De Troyer A. Abdominal muscle use during breathing in patients with chronic airflow obstruction. Am Rev Respir Dis 1992; 146: 16-21.

2. Martinez FJ, Couser JI, Celli BR. Factors influencing ventilatory muscle recruitment in patients with chronic airflow obstruction. Am Rev Respir Dis 1990; 142: 276282.

3. Ninane V, Yernault JC, De Troyer A. Intrinsic PEEP in patients with chronic obstructive pulmonary disease: Role of expiratory muscles. Am Rev Respir Dis 1993; 148: 1037-1042.

4. Yan S, Kaminski D, Sliwinski P. Inspiratory muscle mechanics of patients with chronic obstructive pulmonary disease during incremental exercise. Am J Respir Crit Care Med 1997; 156: 807-813.

5. Grimby G, Goldman M, Mead J. Respiratory muscle action inferred from rib cage and abdominal V-P partitioning. J Appl Physiol 1976; 41: 739-751.

6. Eltayara L, Becklake MR, Colta CA, Milic-Emili J. Relationship between chronic dyspnea and expiratory flow limitation in patients with chronic obstructive pulmonary disease. Am J Respir Crit Care Med 1996; 154: 1726-1734.

7. Haluszka J, Chartrand DA, Grassino AE, Milic-Emili J. Intrinsic PEEP and arterial $P \mathrm{a}_{1} \mathrm{CO}_{2}$ in stable patients with chronic obstructive pulmonary disease. Am Rev Respir Dis 1990; 141: 1194-1197.

8. Dal Vecchio L, Polese G, Rossi R, Rossi A. Intrinsic positive end-expiratory pressure in stable patients with chronic obstructive pulmonary disease. Eur Respir $J$ 1990; 3: 74-80.

9. Dodd DS, Brancatisano T, Engel LA. Chest wall mechanics during exercise in patients with severe chronic airflow obstruction. Am Rev Respir Dis 1984; 129: 33-38.

10. Lessard MR, Lofaso F, Brochard L. Expiratory muscle activity increases intrinsic positive end-expiratory pressure independently of dynamic hyperinflation in mechanically ventilated patients. Am J Respir Crit Care Med 1995; 151: 562-569.

11. Yan S, Kayser B, Tobiasz M, Sliwinski P. Comparison of static and dynamic intrinsic positive end-expiratory pressure using the Campbell diagram. Am J Respir Crit Care Med 1996; 54: 938-944.

12. Zakynthinos SG, Vassilakopoulos T, Zakynthinos E, Roussos C, Tzelepis GE. Correcting static intrinsic positive end-expiratory pressure for expiratory muscle contraction. Am J Respir Crit Care Med 1999; 160: 785-790.
13. Beck J, Sinderby C, Lindström L, Grassino A. Effects of lung volume on diaphragm EMG signal strength during voluntary contractions. J Appl Physiol 1998; 85: 11231134.

14. American Thoracic Society. Lung function testing: selection of reference values and interpretative strategies. $\mathrm{Am}$ Rev Respir Dis 1991; 144: 1201-1218.

15. Sinderby C, Beck J, Spahija J, Weinberg J, Grassino A. Voluntary activation of the human diaphragm in health and disease. J Appl Physiol 1988; 85: 2146-2158.

16. Sinderby C, Navalesi P, Beck J, et al. Neural control of mechanical ventilation in respiratory failure. Nat Med 1999; 5: 1433-1436.

17. Beck J, Sinderby C, Weinberg J, Grassino A. Effects of muscle-to-electrode distance on the human diaphragm electromyogram. J Appl Physiol 1996; 79: 975-985.

18. Sinderby CA, Beck JC, Lindström LH, Grassino A. Enhancement of signal quality in esophageal recordings of diaphragm EMG. J Appl Physiol 1997; 82: 1370-1377.

19. Parthasarathy S, Jubran A, Tobin MJ. Cycling of inspiratory and expiratory muscle groups with the ventilator in airflow limitation. Am J Respir Crit Care Med 1998; 158: $1471-1478$.

20. Zakynthinos S, Vassilakopoulos T, Zakynthinos E, Roussos C. Accurate measurement of intrinsic positive end-expiratory pressure: how to detect and correct for expiratory muscle pressure. Eur Respir J 1997; 10: 522529.

21. Appendini L, Patonio A, Zanaboni S, et al. Physiologic effects of positive end-expiratory pressure and mask pressure support during exacerbations of chronic obstructive pulmonary disease. Am J Respir Crit Care Med 1994; 149: 1069-1076.

22. Begin P, Grassino A. Inspiratory muscle dysfunction and chronic hypercapnia in chronic obstructive pulmonary disease. Am Rev Respir Dis 1991; 143: 905-912.

23. Beck J, Sinderby C, Lindström L, Grassino A. Crural diaphragm activation during dynamic contractions at various inspiratory flow rates. $J$ Appl Physiol 1998; 85: 451-458.

24. Campbell EJM. The inspiratory muscles and the mechanics of breathing. London, Lloyd-Luke, 1958.

25. Yan S, Sliwinski P, Gauthier AP, Lichros I, Zakynthinos S, Macklem PT. Effect of global inspiratory muscle fatigue on ventilatory and respiratory muscle responses to $\mathrm{CO}_{2}$. J Appl Physiol 1993; 75: 1371-1377.

26. Macklem PT, Gross D, Grassino A, Roussos C. Partitioning of the inspiratory pressure swings between diaphragm and intercostal/accessary muscles. J Appl Physiol 1978; 44: 200-208.

27. Kayser B, Sliwinski P, Yan S, Tobiasz M, Macklem PT. Respiratory effort sensation during exercise with induced expiratory flow limitation in healthy humans. $J$ Appl Physiol 1997; 83: 936-947. 